\title{
Real-time water quality monitoring system: an IoT application
}

\author{
Zulkhairi Mohd Yusof, Md Masum Billah, Kushsairy Kadir \\ Universiti Kuala Lumpur British Malaysian Institute (UniKL BMI), Malaysia
}

\begin{tabular}{|c|c|}
\hline Article Info & ABSTRACT \\
\hline Article history: & There is huge number of diseases which is caused through water drinking \\
\hline Received Aug 21, 2018 & $\begin{array}{l}\text { that being polluted was supplied to the consumer. This is something that } \\
\text { cannot be underestimate because it can cause high cost of treatment or death. }\end{array}$ \\
\hline Revised Nov 18, 2018 & It's not only causes troubles to the consumer which is human, it is also \\
\hline Accepted Mar 10, 2019 & $\begin{array}{l}\text { included all aquatic life and surrounding ecosystem. There is way to } \\
\text { overcome this problem that created before which is take the sample of water }\end{array}$ \\
\hline Keywords: & $\begin{array}{l}\text { to the lab. The result of water being polluted or not will be released by the } \\
\text { lab. But this system going to take lot of times because there are few }\end{array}$ \\
\hline IoT & processes that should be followed. This system is not suitable anymore \\
\hline Real-time & $\begin{array}{l}\text { because it is not portable, easy and fastest. It should be upgraded to the loT } \\
\text { system because it can cut times, internet base and people will be more alert to }\end{array}$ \\
\hline Water quality & $\begin{array}{l}\text { the quality of water. Therefore, a real-time water quality monitoring system } \\
\text { is proposed in this research to reduce number of fatalities happened } \\
\text { from water. }\end{array}$ \\
\hline
\end{tabular}

Copyright @ 2019 Institute of Advanced Engineering and Science. All rights reserved.

Corresponding Author:

Md Masum Billah,

Universiti Kuala Lumpur British Malaysian Institute (UniKL BMI),

Kuala Lumpur, Malaysia.

Email: drmdmasum@unikl.edu.my

\section{INTRODUCTION}

Internet of Things is not something new in technology area. The term "Internet of Things"(IoT) itself was first used 1999 by British technology pioneer Kevin Ashton to describe a system in which objects in the physical world could be connected to the Internet by sensors [1]. It also can be marked as medium of communication between people with things and things with things. It is develop widely due to the advanced Wireless Technology. IoT was created to fulfill the objective which is any time, anyone and any place. There are lots of application that used IoT on defined areas such as Building and home automation, Smart cities, Smart manufacturing, Automotive, Wearables and health care. All of these areas are trusted to have high potential for exponential growth.

The IoT means the Internet environment of generating, mutually collecting, sharing, and using information by allowing all the things, such as people, around things, data, etc, to be connected to wired and wireless networks [2]. With this, consumers have extended the scope of connection to the virtual world by communicating with each other widely and gain knowledge about how the things networked. Then, the recent advance in high-speed wireless technologies has accelerated along with the supply of connected devices based on networks such as smartphones, tablet PCs, e-book devices, etc [3].

The WHO (world health organization) estimated, in India among 77 million people is suffering due to not having safe water. WHO also estimates that $21 \%$ of diseases are related to unsafe water in India. Also, more than 1600 deaths alone cause due to diarrhea in India daily [4]. Therefore, water quality monitoring is essential for everyday life. Currently internet of thing (IoT) technology is very popular for realtime systems. The internet of thing is computing concept that describes a future where every day physical object will be connected to the internet and be able to identify themselves to other devices [5]. IoT often refers to a large network of sensor-enabled devices designed to collect data about their environment, which frequently includes data related to people. This data presumably provides a benefit to the device's 
owner, but frequently to the device's manufacturer or supplier as well [6]. In addition, some other technology is also used for water quality monitoring system [7-16].

In many countries, they still use old and traditional techniques, where the farmer or healthy society which responsible on water quality will visits the fond or the specific areas to monitor and control the water quality manually. They take the water sample to the lab in order to know the values of the water quality parameters and then take appropriate controlling measures. This entire process is tiresome, lengthy and costly due to many process involved. In order to improve the method, they should continuously monitor for some critical parameters and maintain the records. This requires the system to be automated and new which is more innovative technology has to be used to simplify the maintenance complexity, fasten the process and finally earn good profit.

The aim of this paper is to discuss the development application of IoT in the area of healthy monitor specialty on water quality. The device not only displays the result of water quality but will detect the temperature as well. This device was started with detect the surrounding temperature and display the temperature reading on the LCD. When the temperature part was success, the next sensor was attached to the project which is water quality sensor will operate. This sensor will give result on quality of water either it is clean or not. When it is stated clean, means the water unpolluted and save to consume by the consumer.

\section{MATERIALS AND METHODS}

This paper will present the quality of water at certain place. The result of water quality will be presented in month range. This chapter will show the proposed system used in order to obtain the results. It also will explain in details on how the system or process involved in the project. in this project, there are two parts that included which is hardware and software.

\subsection{Hardware and Software Design}

The components that used in the project are Launchpad, LCD and Sensor. Launchpad CC3200 consist of application Microcontroller, Wi-Fi Network Processor and Power Management Subsystem. It is the latest ARM Cotex-M4 80MHz processor with built-in Wi-Fi. Since the project was internet based, it is suitable to use this device due to its specialty. LCD 430BOOST SHARP96 was used to display the result that obtained from the sensor. The sensor that used will detect the water quality and the result will be display on the LCD. The LCD and sensor will attach to the Launchpad according to the pin on the Launchpad that can be referred from the datasheet and the devices will be connected to the laptop by using cable. Lastly, $\mathrm{Ph}$ sensor was used to detect the ph value of the water which will display the data on the serial monitor of the Energia software. The data will directly send to the MQTTlens in order to subcribe and publish the data. The flow of the project will be create in the node.red in order to make people surrounding able to get the data through their smart phone. MQTTlens and node.red will be explain on the software design.

Energia software was used to write the coding and give the instruction to the specific device or components. When the devices connected to the laptop, coding will be typed on the Energia software in order to display the results on the LCD. The other part software in this project is Cloud server. Huge numbers of systems are connected in public or private networks, to provide energetically scalable infrastructure for application, data and file storage. With built-in Wifi on CC3200, it send out data to the cloud and also subscribe to the data from the cloud. It also quick upgrade to the display so that the data will be better represented. After that, MQTT lens server will be used to publish and subscribe the data. The data can be obtained by the others easily. In short, Energia (for programming CC3200), MQTT Lens (to check the cloud setup), node.js (to setup webserver for node-red), node-red (for programming the user interface in webserver as well as smart phone). The application can be downloaded the smart phone and user can store the message on the phone anytime and anywhere.

Figure 1 describes on how the flow of sending and receiving data from cloud. This step was continued from previous step. MQTT (picture 3.7) is a medium that will allow us to subscribe to the eclipse server separately. It was used to see what the data is on the MQTT server that's arriving there and to be able to send a publication to it for the high and low setpoint to see if the CC3200 is going to receive it. MQTT also provides messaging qualities of service with three different level as in table below.

Next, Node Red which is an application that runs under Windows to subscribe the data and give us a nice little UI display that we can use on smart device. In short, Energia (for programming CC3200), MQTT Lens (to check the cloud setup), node.js (to setup webserver for node-red), node-red (for programming the user interface in webserver as well as smart phone). The application can be downloaded the smart phone and user can store the message on the phone anytime and anywhere. 


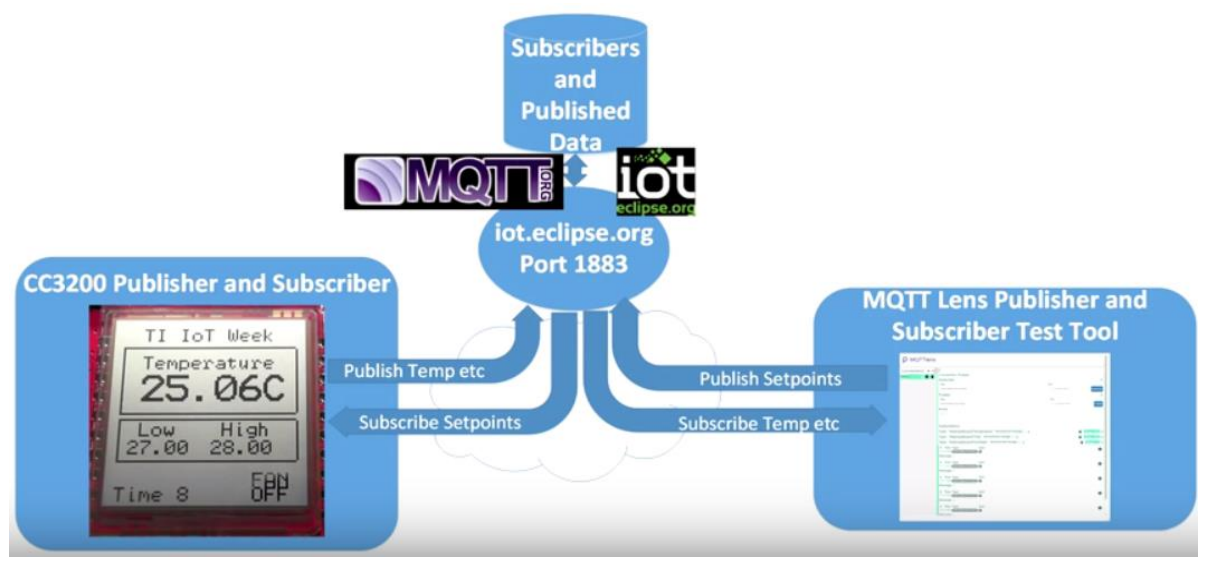

Figure 1. The process describing sending and receiving data from the cloud

\subsection{Procedure}

The quality of water in the specific river will be taking for a month by using the device. The Cloud server and MQTTlens were used as a medium to store, publish and subscribe the data as shown in Figure 2. After draw the flow of the project, the data will be display nicely on the laptop. People surrounding also can get the data through their smart phone same as the data display on the laptop. This is to monitor the quality of water at that specific area.

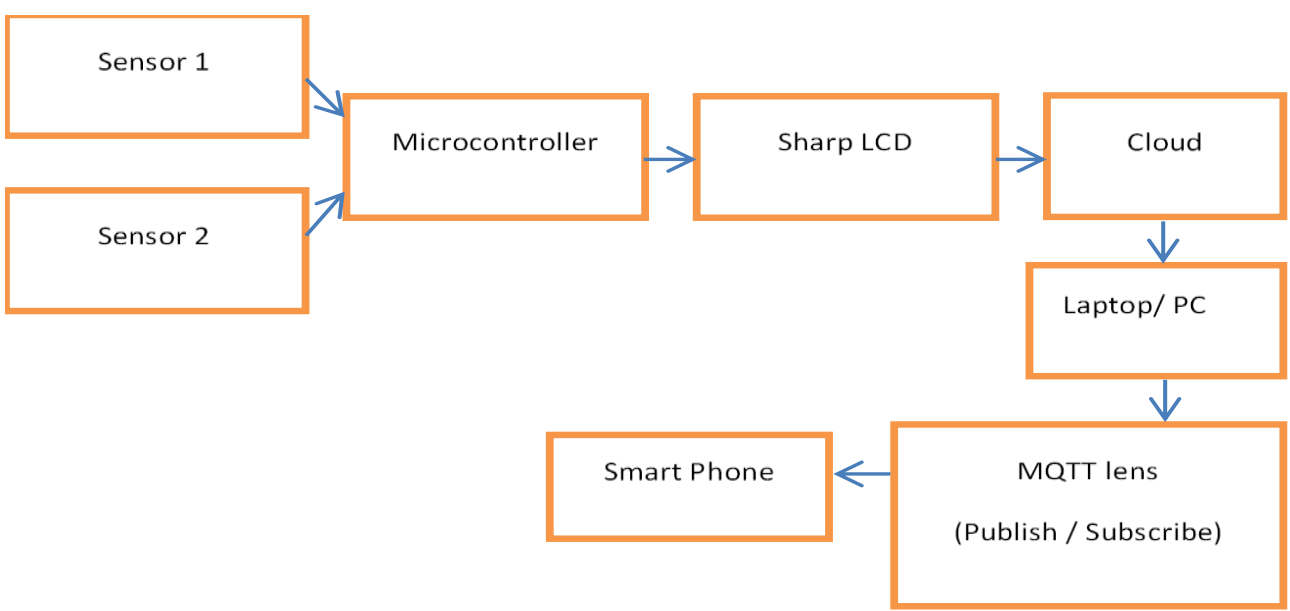

Figure 2. Block diagram of the process to collect data

\section{RESULTS AND DISCUSSION}

\subsection{Project Implementation}

The ph sensor that had been connected to the Launchpad cc3200 which connected by the connector will be submerged on the solution with specific ph level. The basic principle of ph meter is to measure the concentration of hydrogen ions. Acid dissolved in water forming positively charged hydrogen ion $(\mathrm{H}+)$ as shown in Figure 3. In short, the greater the concentration of hydrogen ion, the stronger the acid level. Similarly alkali or bases dissolve in water forming negatively charge hydrogen ion (OH-). The stronger a base is the higher concentration of negatively charge of hydrogen ion. It will determine the ph. $\mathrm{Ph}$ meter made up of probe which itself made up of 2 electrodes. This probe passes the electrical signal. At the end of the probe, there are bulb that should be submerged on the solution which will detect the ph level. If the bulb was broke or did not submerged on the solution, the ph level will not detected. As principle stated above, the ph meter will be detected and the ph level will be display on the serial monitor. It is because, the coding that wrote was gave an instruction to display the data on the serial monitor. Here is the normal range of the ph level and the voltage that detected. 


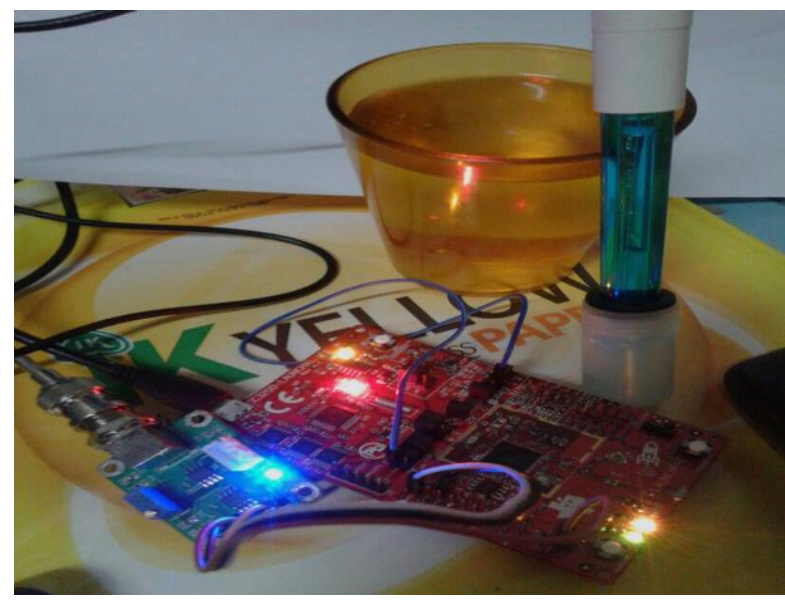

Figure 3. Model of the project

The process flow in shown in Figure 4. After connected to the MQTTlens, it will come out the subscribe and publish page. With that, we need to fill up the subscribe and publish topic just like wrote on the coding. After fill up the topics, the data will display same as on the serial monitor. The flow of the project will be draw on the node.red. The results of the real-time monitoring system were viewed on the MQTTlens dashboard that was installed on the google chrome as shown in Figure 5. After click the save changes button, it will connect the project and will display the topic to publish and subscribe in order to monitor the data same as display on the serial monitor. After draw the flow, the data will display nicely as in Figure 5. With that, people surrounding can get the data through their smartphone same as shown on Figure 5.
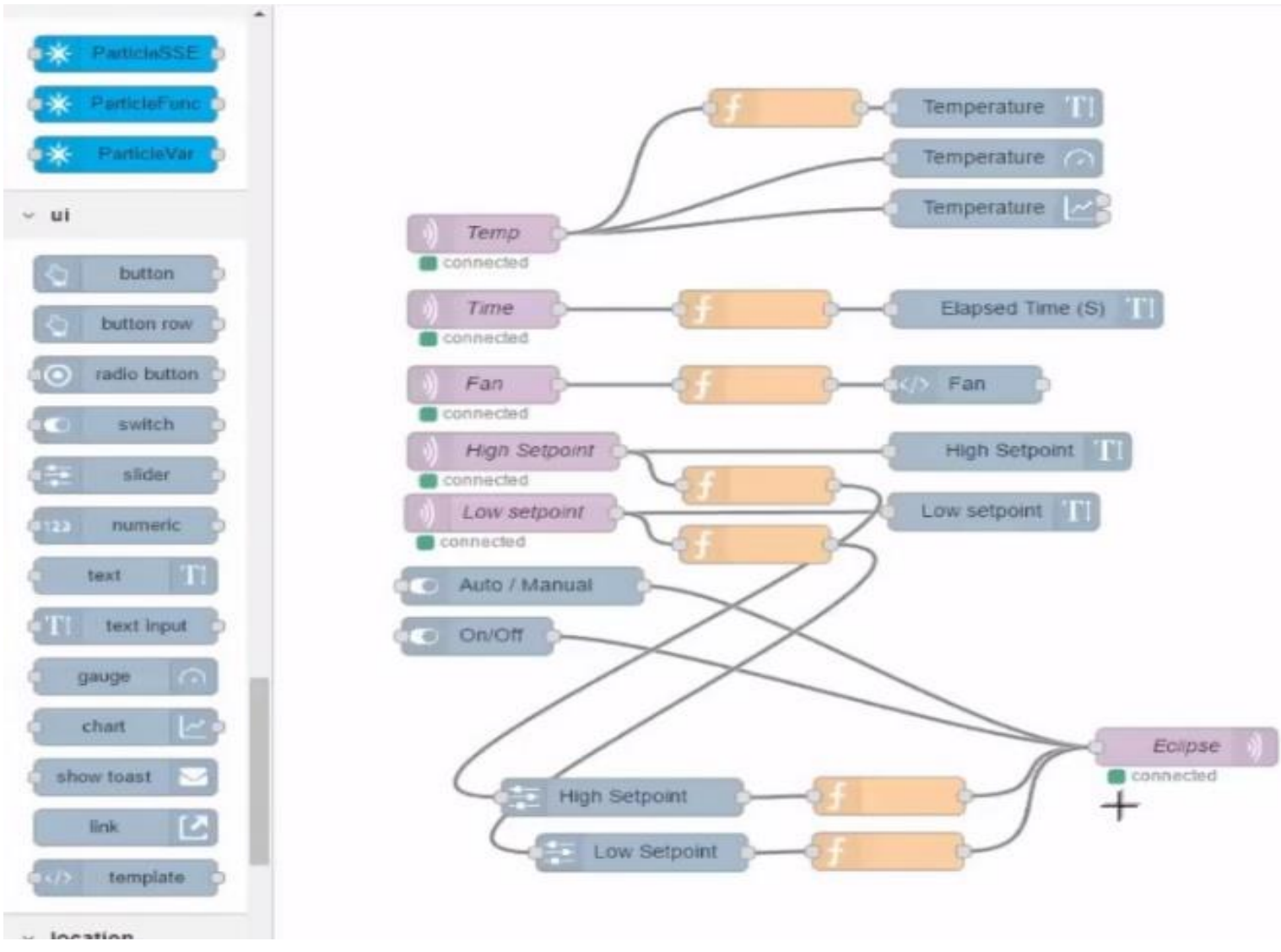

Figure 4. The flow of the program 


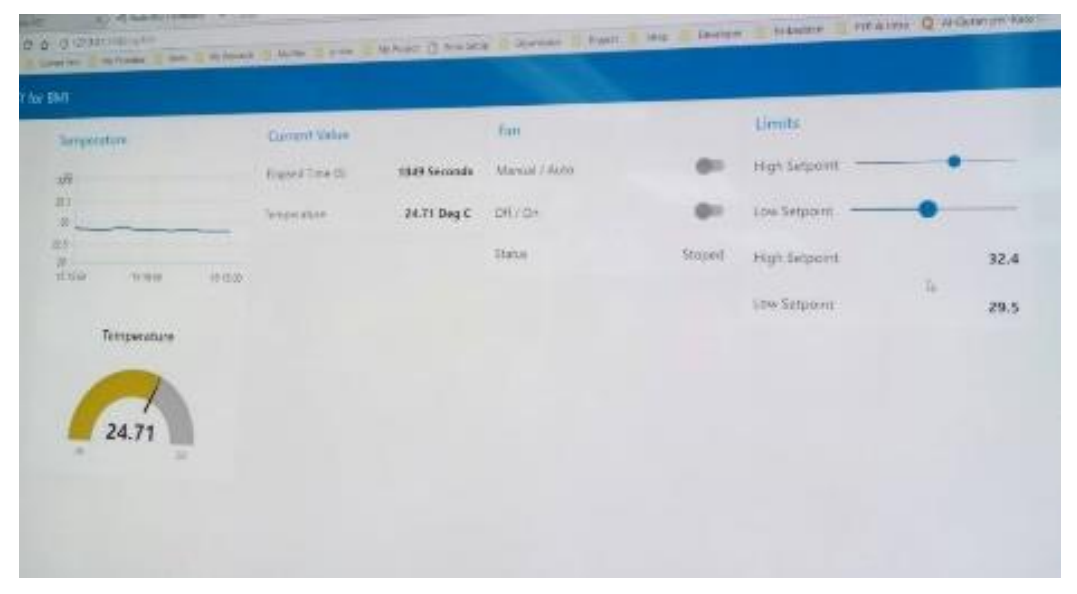

Figure 5. Dashboard display on laptop and smart phone

\section{CONCLUSIONS}

As a conclusion, it can conclude as this project meet the objective that being proposed for this system. One of the aims is to make people surrounding more alert with the water quality because it supposed to be internet-based project and informally make people easier to get the data anytime and anywhere. This research has covered more on how to develop the Internet of Thing which is technology or system that highly developing in the world. There are few stages that involved in the Internet of Thing (IoT) for real-time operation. It can make it easier to people that responsible on the quality of water that will make everyone more alert with the quality of water that they took every day. This system also did not used high cost if compare with the current system used. It can be considered as low-cost system that give advantage to everyone.

\section{REFERENCES}

[1] Young-Mo Kang, Mi-Ran Han, Kyeong-Seok Han, Jong-Bae Kim, “A Study on the Internet of Things (IoT) Applications", IJSEIA, Vol 9, 2015.

[2] Meenakshi, I. K., and Sudha George. "Cloud server storage security using TPA." Int. J. Adv. Res. Comput. Sci. Technol 2.1 (2014): 295-299.

[3] Beena.V, Mr. KhajaMoinuddin, "Water Quality Measurement and Control from Remote Station for Pisiculture Using NI myRIO”, Vol 2, 2015.

[4] Jayti Bhatt, Jignesh Patoliya, "Iot Based Water Quality Monitoring System", 2016.

[5] Poonam J. Chavan, Manoj A. Mechkul, "IoT Based Water Quality Monitoring”, 2016.

[6] An Overview "Understanding the Issues and Challenges of a More Connected World", 2015.

[7] Pandian D R, Dr. Mala K, "Smart Device to monitor water quality to avoid pollution in IoT environment", Vol 12, 2015.

[8] Mr.Kiran Patil, Mr.Sachin Patil, Mr. Sanjay Patil and Mr.Vikas Patil, “ Monitoring of Turbidity, ph \& Temperature of Water Based on GSM", Vol 2, 2015.

[9] Peng Jiang, Hongbo Xia, Zhiye He, Zheming Wang, "Design of a Water Environment Monitoring System Based on Wireless Sensor Networks", 2009.

[10] Akanksha Purohit, Ulhaskumar Gokhale, "Real Time Water Quality Measurement System based on GSM", Vol 9, 2014.

[11] Texas Instruments Sharp LCD BoosterPack (430BOOST-SHARP96) for the Launchpad journal, 2014.

[12] Shailaja.M.Gunda Nikkam, Prof. Dr. V. R. Pawar, "Analyzing Water Quality For Industrial Application Under Iot Environment", Vol 3, 2016.

[13] Tito Yuwono, Luqman Hakim, Irfan Ardi, Umar, “The Application of Internet of Things System for Water Quality Monitoring", Vol 8, 2016.

[14] Atlas Scientific Environmental Robotics, Vol 2.6, 2017.

[15] Manpreet Kaur Khurana, Rajesh Singh, Anshuman Prakash, Rohit Chhabra, "An IoT Based Water Health Monitoring", 2016.

[16] Texas Instruments CC3200 SimpleLink WiFi and Internet-of-Things Solution, a Single-Chip Wireless MCU, 2015. 